\title{
Translational Research to Generate Evidence for Rational and Efficient Introduction of New Vaccines in Developing Countries: The Experience of the International Vaccine Institute
}

\author{
John Clemens \\ International Vaccine Institute, Seoul, Korea
}

\section{Key Words}

Translational research $\cdot$ Vaccine introduction - Developing countries

\begin{abstract}
Introduction of new vaccines into affluent countries has occurred at a breathtaking pace in recent years. By comparison, few new-generation vaccines have been introduced into public health programs for the poor in developing countries, and, for those that have, introduction has been painfully slow. Limited financial resources have retarded the introduction. However, the slow pace also stems from a dearth of evidence needed for rational policy decisions. Inadequate support to conduct phase 1 studies of vaccine candidates targeted against diseases of developing countries is a wellknown obstacle. Additionally, other types of translational research are needed to generate the necessary evidence for policy. Many vaccines have been shown to perform less well in impoverished populations in the developing world than in persons residing in more affluent countries. Consequently, phase 2 and phase 3 trials are a second essential type of translational research needed for the introduction of vaccines in developing countries. Moreover, even for vaccines that achieve licensure via clinical trials in developing countries, doubts may remain about whether the burden of disease warrants vaccine introduction, whether the adminis-
\end{abstract}

tration of the vaccine in public health programs will be cost-effective, whether vaccine introduction will be programmatically feasible and acceptable, and whether the introduction of the vaccine will be financially sustainable. To address these residual doubts, a third type of translational research is needed. Since its inception in 1987, the International Vaccine Institute, an international, non-profit research and development organization located in Seoul, Korea, has conducted translational research on new vaccine introduction for a variety of diseases in 22 countries in Asia, Africa and Latin America. In this paper, we describe translational research projects undertaken by the International Vaccine Institute and lessons learned about strategies to increase the impact of translational research on vaccine policy for the developing world.

Copyright $\odot 2008$ Nestec Ltd., Vevey/S. Karger AG, Basel

\section{Introduction}

We live in the midst of a revolution in vaccine development, in which vaccines have been developed and licensed at an unprecedented pace, and new technologies are being brought to bear on the development of new vaccines, including those against diseases which in the past were not considered vaccine preventable. Despite this, the pace of introduction of new and improved vaccines into

\section{KARGER \\ Fax +4161306 1234 \\ E-Mail karger@karger.ch}

www.karger.com
(C) 2008 Nestec Ltd., Vevey/S. Karger AG, Basel 0517-8606/08/0662-0081\$24.50/0

Accessible online at:

www.karger.com/ane
John Clemens, MD

International Vaccine Institute

Kwanak PO Box 14

Seoul 151-600 (Korea)

Tel. +82 2881 1011, Fax +82 2872 2803, E-Mail jclemens@ivi.int 
the public health programs of developing countries has been painfully slow [1]. This is most unfortunate since many of these new-generation vaccines, such as those against diarrhea, meningitis and pneumonia, are targeted against diseases that are major public health problems in developing countries.

Much has been written about the scientific challenges of vaccine discovery and about strategies to improve the process of discovering new vaccine candidates. The financial hurdles and programmatic obstacles that impede the introduction of new vaccines in developing countries are also well known. Less appreciated is the fact that, even for existing vaccine candidates, there may be other formidable scientific challenges that can impede their introduction into public health programs. The term 'translational research' encompasses the research agenda that addresses these challenges. As such, translational research can be thought of as research to translate both experimental vaccine candidates and already licensed vaccines into practical tools that are used in public health practice.

Translational research has been a major focus of the International Vaccine Institute (IVI) in Seoul, Korea. The IVI began in the early 1990s as a seed project of the United Nations Development Programme. At that time, the United Nations Development Programme reasoned that the world was in need of an international research organization devoted exclusively to developing and deploying new vaccines for developing countries. In 1997, the IVI became an autonomous international organization, under the Vienna Convention, and now has 40 countries and the World Health Organization as signatories to its charter, and with the Republic of Korea as the host country. While no longer a UN organization, the IVI remains a member of the UN family of organizations. In this paper, we first delineate the types of studies that fall under the rubric of translational research and then illustrate these studies with work that has been done by the IVI over the past several years.

\section{The Clinical Paradigm for Evaluating Vaccines for Licensure}

To place the rationale for translational research into context, it is helpful to describe the phases of the clinical evaluation of vaccine candidates prior to licensure $[2,3]$. The initial human studies of a candidate that has earlier yielded promising results in preclinical studies are termed phase 1 trials. These trials are typically small in size (e.g., approximately 10-20 subjects), enroll healthy adult subjects and are designed primarily to evaluate whether the vaccine is associated with frequent adverse effects. Phase 1 trials typically evaluate other vaccine effects, such as immune responses and vaccine excretion (for live vaccine candidates), and may be used to determine an optimal dose and regimen for the vaccine candidate.

Candidates found to be suitable in phase 1 trials may then be tested in phase 2 trials. Phase 2 trials are distinguished by their larger sample sizes (often in the range of several hundred subjects), by their ability to evaluate, in a statistically meaningful fashion, immune responses as well as relatively uncommon adverse reactions, and by the eventual inclusion of subjects in the populations and age groups that will be the ultimate targets for the vaccine in public health practice. Additionally, phase 2 trials may measure excretion and transmissibility of live vaccines. Although phase 2 trials are not typically designed to measure vaccine protection against the target infection, special types of phase 2 trials, sometimes termed 'phase $2 \mathrm{~b}$ trials', are conducted to measure vaccine protection [4]. Phase $2 b$ trials refer to 2 different types of studies. In the first, vaccine protection is measured against experimental challenges with the target pathogen. In the second, vaccine protection is measured under field conditions, but with sample sizes smaller than those typically required by phase 3 trials. Both types of phase $2 \mathrm{~b}$ studies are undertaken to establish a proof of principle that the vaccine can protect against disease in humans, before investing in more expensive and logistically demanding phase 3 trials.

Finally, for candidates found to be suitably safe and immunogenic in phase 2 trials, phase 3 trials may be mounted. The distinctive role of phase 3 trials is that they measure vaccine protection against naturally occurring infections in populations at risk. Phase 3 trials are usually quite large, often enrolling thousands of subjects, and are always conducted in the populations and age groups to be targeted by the vaccine. Because of their large size, phase 3 trials usually are capable of detecting rather uncommon adverse events associated with vaccination. Moreover, because phase 3 trials measure both immune responses to vaccination and vaccine protection, they may be able to correlate the two, and thus, to derive immunological correlates of protection. Phase 1 trials may be conducted in a controlled or uncontrolled fashion; phase 2 and phase 3 trials conventionally employ double-blind, randomized, controlled trial designs. 


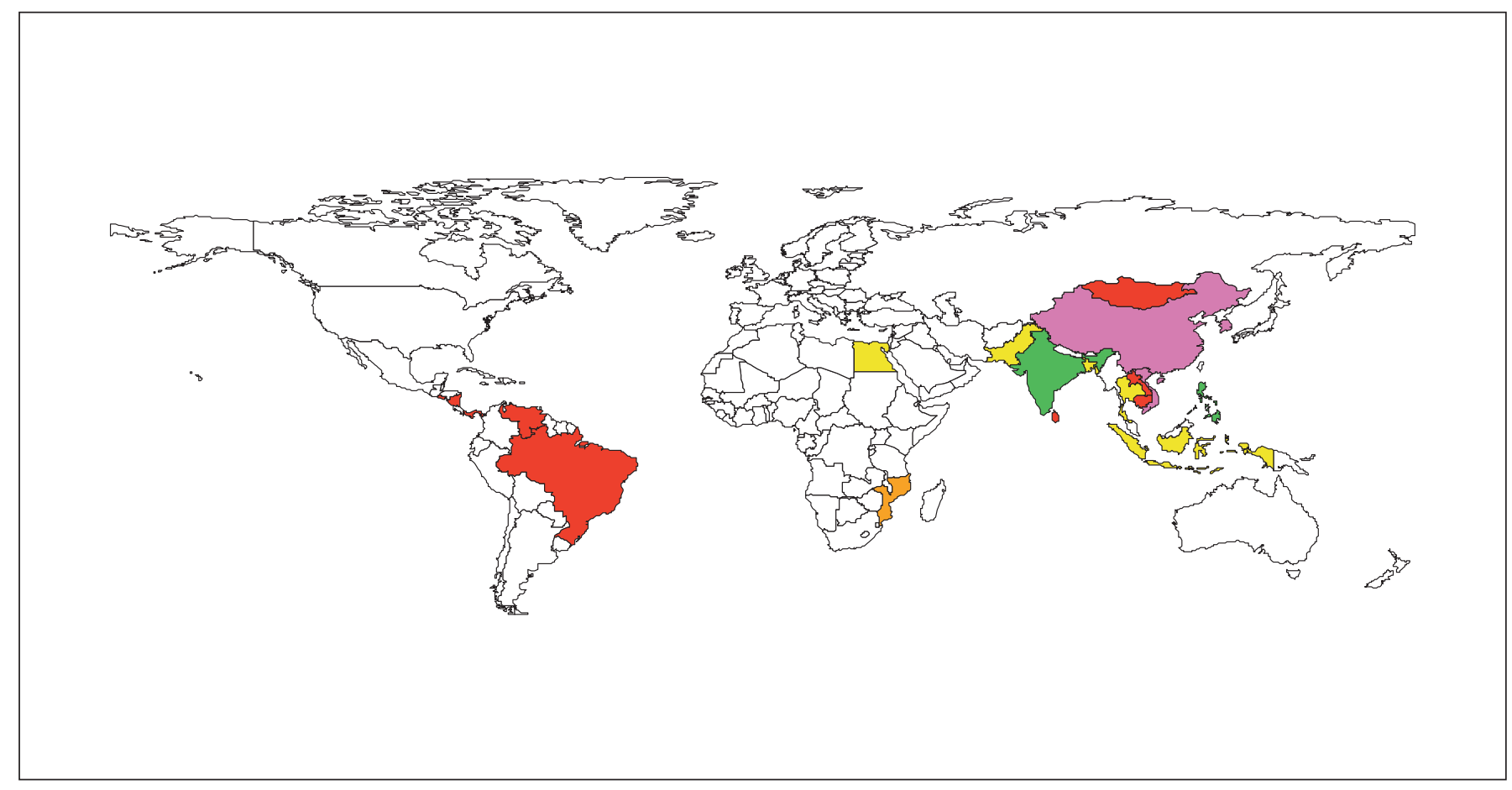

Fig. 1. Geographical locations of the IVI translational research programs.

\section{Translational Research: Overcoming Hurdles to the Introduction of New Vaccines}

Translational research to accelerate the introduction of a vaccine candidate into public health programs in developing countries addresses 3 types of scientific challenges. First, a vaccine candidate may languish on the laboratory shelf without an opportunity to be tested in humans. This developmental impasse is most notorious for orphan vaccines, targeted to diseases that are of little or no interest to populations in industrialized countries and having limited potential profitability for vaccine producers. Examples of such vaccines include those directed against leishmaniasis, hookworm disease and schistosomiasis. Because such diseases primarily affect developing countries and because affluent travelers to developing countries are not at a particularly high risk of contracting these diseases, there is little economic incentive for industry to undertake expensive clinical development programs.

Second, even for vaccines that are of interest to populations in industrialized and less developed countries alike, there may be interest in and funding for human studies in industrialized countries only, where the most profitable markets are located. The conduct of parallel clinical development pathways in both industrialized and developing countries may impose risks of substantial delays in licensure in markets of industrialized countries, with major loss of income generation for industry. This creates a problem for developing countries because the results of studies done in populations of industrialized countries do not always predict the performance of a vaccine in populations of developing countries. Such has been the case for early-generation vaccines against rotavirus [5-7] and against Haemophilus influenzae type b (Hib) [8-10], which were highly protective in children of industrialized countries but poorly protective in children of developing countries. Too often in the past, studies have been done in developing countries many years after the licensure of a vaccine in industrialized countries, creating an unacceptable delay in vaccine introduction even when such trials were successful.

Third, even if a vaccine has been shown to be safe and protective in phase 3 trials in developing countries, policymakers may still feel uncertain about whether an adequate case can be made for introducing the vaccine into public health programs in their countries. This is because 
the evidence provided by pre-licensure evaluations, even those done in developing countries, typically fails to address many practical questions about implementing a new vaccine in real-life programs, which must be answered before a decision can be made to introduce a vaccine $[11,12]$. The insufficiency of evidence from the required downstream research constitutes the third scientific hurdle.

Overcoming these 3 hurdles requires 3 types of translational research [11-13]. The first scientific hurdle requires the conduct of initial human studies (phase 1) of promising vaccine candidates. The second requires phase 2 and phase 3 studies to achieve vaccine licensure in developing country settings, ideally conducted in parallel with evaluations in industrialized countries. And the third hurdle requires, for vaccines that have proven safe and effective in phase 3 trials in the developing world, generating epidemiological, clinical, economic, behavioral and policy evidence needed to make an adequate case that the introduction of a new vaccine into public health programs in a developing country is rational, feasible, acceptable and affordable.

Table 1 shows the current disease priorities for the IVI translational research on new generation vaccines, and figure 1 provides a geographical picture of the countries with which the IVI has partnered to conduct its translational research programs. In the remainder of this paper, we provide examples from the translational research programs of the IVI to illustrate how research has been undertaken to address the challenges of overcoming these hurdles.

\section{Translational Research on Vaccines before Licensure: Partnerships to Assure Vaccine Access}

Generation and communication of evidence gained by translational research are critical to rational policy decisions about vaccine introduction and can act as a 'pull' mechanism to create demand for a vaccine and funding for vaccine purchase [12]. Yet, this research will not have an impact on vaccine introduction unless it is coupled to strategies which make the targeted vaccine available in sufficient quantities and at cost-competitive prices. Public-private partnerships between non-profit entities such as the IVI and vaccine manufacturers have become a common mechanism whereby the risks of vaccine development before licensure are shared between the public and private sectors, and the public sector can thereby benefit by obtaining assurance of an ample and cost-
Table 1. Priorities of the IVI in translational research

1. Vaccines against enteric infections

Rotavirus

Enterotoxigenic Escherichia coli

Shigellosis

Cholera

Typhoid fever

2. Vaccines against causes of meningitis and/or pneumonia $\mathrm{Hib}$

Pneumococcus

Meningococcus

Influenza

Tuberculosis

3. Vaccines against flavivirus infections

JE

Dengue fever

competitive supply of the vaccine when and if the vaccine is licensed.

The IVI has pursued several different models for such collaborations, all done within the translational research programs. All of these models entail working with vaccine producers committed to making a vaccine available in sufficient supply and at cost-competitive prices once the vaccine becomes licensed. One model entails collaboration with major international pharmaceutical companies. For example, the IVI is currently collaborating with Merck Vaccines on a phase 3 trial of a live oral vaccine against rotavirus (Rotateq ${ }^{\mathrm{TM}}$ ). This vaccine has been licensed on the basis of an extensive series of clinical trials done in industrialized countries [14]. However, because live oral vaccines against rotavirus and other pathogens have performed less well in children living in developing countries, the GAVI Alliance (the major procurer of vaccines for children in developing countries) will purchase this vaccine for developing countries only after data on the efficacy of this vaccine have been obtained from clinical trials in the developing world. With sponsorship of the Rotavirus Vaccine Program at PATH (Program for Appropriate Technology in Health) and Merck, the IVI is now working with the National Institute of Hygiene and Epidemiology of Vietnam on a phase 3 efficacy trial of this vaccine in Nha Trang, Vietnam. The trial will complement several others being done in developing countries in Asia and Africa.

A second model of collaboration entails work on a vaccine candidate currently held by a biotechnology company in the industrialized world, with the aim of ultimate technology transfer to a company capable of producing the vaccine at large scale and at affordable prices. An example of a vaccine being evaluated in this 
type of collaboration is Peru-15, a genetically attenuated, live oral vaccine against cholera originally developed at Harvard University and subsequently licensed to Avant Immunotherapeutics. Despite promising results in North American volunteers, the vaccine languished for years without evaluation in a cholera-endemic country. In 2000, the IVI launched the Diseases of the Most Impoverished (DOMI) program, supported by the Bill and Melinda Gates Foundation, to accelerate the introduction of new-generation vaccines against typhoid, cholera and shigellosis. As a component of the DOMI program, the IVI launched a series of phase 2 trials of Peru-15 in Bangladesh, in collaboration with investigators at the International Centre for Diarrhoeal Disease Research, Bangladesh. The trials demonstrated robust safety and immunogenicity of a single dose of Peru-15 in Bangladeshi adults, children and infants $[15,16]$. Based on these promising results, and with further support from the Gates Foundation, the IVI is now coordinating a clinical development program for this vaccine in both India and Bangladesh, in collaboration with the National Institute of Cholera and Enteric Diseases and the International Centre for Diarrhoeal Disease Research, Bangladesh, respectively. This program includes additional phase 2 trials, leading to a large phase 3 trial to be conducted in Bangladesh. If the phase 3 trial proves successful, the IVI will assist Avant in transferring the vaccine to a qualified vaccine producer in a cholera-endemic country.

Another example of the second model concerns the live oral vaccine candidate against typhoid fever, ZH09, which has yielded very promising results as a single-dose vaccine in both inpatient and outpatient studies done in the US and the UK [17]. Because of these encouraging results, the IVI collaborated with Emergent Europe in the UK, the developer of this vaccine, and the Wellcome Tropical Disease Unit in Ho Chi Minh City on phase 2 studies of this vaccine in a typhoid-endemic population in Vietnam. Studies in adults and school children revealed acceptable vaccine safety and immunogenicity, and further phase 2 studies and a phase 3 efficacy trial are now being planned in Kolkata, India. As was the case for the Peru-15 vaccine, the IVI will also assist in the transfer of production technology of this vaccine to a producer in a typhoid-endemic country, so that the vaccine can ultimately be made available at affordable prices for public health programs.

A third model of collaboration with the industry in the translational research program 9 of the IVI involves evaluation of a vaccine generated by an emerging vaccine manufacturer, illustrated by the work of the IVI on an orally administered, killed whole-cell (WC) vaccine against cholera. The WC vaccine was transferred 2 decades ago by Professor Jan Holmgren in Sweden to a Vietnamese producer (now called VaBiotech) [18]. The oral WC vaccine has been used in public-health programs of Vietnam for many years, but, because the Vietnamese national regulatory authority is not WHO-approved, the vaccine has not been used outside of Vietnam. The vaccine production specialists of the IVI have worked with VaBiotech to upgrade the quality of production of the vaccine used in Vietnam to meet WHO standards. In addition, to permit the international use of this vaccine in cholera-endemic countries, the IVI team is transferring the production technology for this vaccine to 2 producers in cholera-endemic countries with WHO-approved national regulatory authorities (India and Indonesia). The IVI is also assisting these producers with the design and conduct of phase 2 and phase 3 clinical studies needed for licensure.

A fourth model of collaboration involves development of a vaccine candidate in-house at the IVI, with subsequent transfer of the candidate to an emerging vaccine manufacturer and conduct of clinical studies necessary for licensure. An example of this model concerns Vi polysaccharide-protein conjugate vaccine, developed by Dr. John Robbins and colleagues at the US National Institutes of Health, which has been shown in a proof-of-principle phase $2 \mathrm{~b}$ trial to be highly protective in Vietnamese toddlers [19]. However, despite these promising data, no vaccine producer has developed this vaccine for its product portfolio. In collaboration with the laboratory of Dr. Robbins, the process and formulation group at the IVI has developed a new Vi-diphtheria toxoid conjugate vaccine and has recently embarked upon a program of technology transfer and clinical development to licensure of the vaccine in collaboration with an Indian manufacturer that has made a commitment to the production of the vaccine at affordable prices for public health programs in typhoid-endemic countries.

From these examples, it can readily be appreciated that conducting clinical trials of vaccines in developing countries is but one element of a strategy of achieving access to these vaccines by poor populations in these settings. Sharing the financial and other risks inherent in developing a vaccine is critical to ensuring that a vaccine will be available to public sector programs. The IVI is fortunate in having staff with a broad range of skills, including vaccine development and process research, vac- 
Table 2. Matrix of evidence developed by the DOMI program for the Vi vaccine against typhoid fever

\begin{tabular}{|c|c|c|c|c|c|c|c|}
\hline \multirow[t]{2}{*}{ Type of activity } & \multicolumn{7}{|l|}{ Country } \\
\hline & Bangladesh & China & India & Indonesia & Pakistan & Thailand & Vietnam \\
\hline Prospective studies of the burden of typhoid fever & $\times$ & $\times$ & $\times$ & $\times$ & $\times$ & & $\times$ \\
\hline Meta-analyses of typhoid disease burden data & $\times$ & $x$ & $x$ & $\times$ & $x$ & $\times$ & $x$ \\
\hline $\begin{array}{l}\text { Assessment of feasibility, acceptability and } \\
\text { impact of immunization with the Vi vaccine }\end{array}$ & & $x$ & $\times$ & $\times$ & $\times$ & & $\times$ \\
\hline Cost of typhoid illness studies & & $x$ & $\times$ & $x$ & $x$ & & $x$ \\
\hline Cost of Vi vaccine delivery studies & & $\times$ & $\times$ & $\times$ & $\times$ & & $x$ \\
\hline $\begin{array}{l}\text { Cost-effectiveness analyses of } \\
\text { immunization with the Vi vaccine }\end{array}$ & & $\times$ & $x$ & $\times$ & $\times$ & & $x$ \\
\hline $\begin{array}{l}\text { Assessment of demand/willingness to pay } \\
\text { for the Vi vaccine }\end{array}$ & & $\times$ & $\times$ & $\times$ & $x$ & & $x$ \\
\hline Policy analyses of Vi vaccine introduction & $x$ & $x$ & $\times$ & $\times$ & $\times$ & $\times$ & $x$ \\
\hline
\end{tabular}

cine technology transfer, conduct of clinical trials, formulation of regulatory strategies, and business negotiations. This wide array of talents gives the IVI a high degree of flexibility to undertake public-private partnerships that complement its translational research programs.

\section{Translational Research on Vaccines after Licensure: An Example}

It may seem odd that translational research is needed to address the third scientific hurdle for vaccines that have completed phase 3 trials and are already licensed. However, there is a very long list of vaccines that are potentially useful for developing countries, are licensed and used in developed countries, but are not widely used in public health programs in developing countries. The list includes, among others, measles-mumps-rubella vaccine, newer-generation typhoid and oral cholera vaccines, and pneumococcal and Hib conjugate vaccines. The reasons for this disparity in use of these vaccines between developed and developing countries are several and include the limited finances available for the purchase of vaccines for poor countries. Another important reason is a dearth of evidence in developing countries for the burden and costs of the diseases targeted by these vaccines, the feasibility and acceptability of introducing these vaccines into real-life public health programs, the costs and cost-effectiveness of using the vaccines pro- grammatically, and the practical preventive impact of the vaccines to be expected under programmatic conditions.

One example of an underutilized vaccine that could be of great benefit to developing country populations is the Vi polysaccharide vaccine against typhoid fever. Despite evidence that typhoid fever kills several hundred thousand people annually in the developing world [20], the use of typhoid vaccines in developing countries is rare. This is particularly frustrating in view of the availability of 2 newer-generation vaccines that have been licensed for many years - oral Ty21a and injectable Vi polysaccharide. Vi polysaccharide is particularly attractive for developing countries because it is given in a single dose, it is relatively thermostable, and it is licensed by multiple vaccine producers, both multinational companies and companies in the developing world. Importantly, several of the latter manufacturers produce this vaccine cheaply (approximately USD 0.50 per dose) and at high quality.

As a part of the DOMI program, the Vi polysaccharide vaccine was selected for accelerated introduction in view of its desirable features for use in developing countries, cited earlier. Table 2 shows the research studies conducted on Vi vaccine and on typhoid fever in the DOMI partner countries. Countries judged to be early adopters received the full complement of studies judged necessary to inform policymakers on the introduction of $\mathrm{Vi}$.

The results of these studies [21-39] demonstrated that typhoid fever is a major problem, especially in the 
study populations in South and Southeast Asia, that the $\mathrm{Vi}$ vaccine could be feasibly delivered in both schooland community-based programs, that the cost-effectiveness of the Vi vaccine made it a 'best buy', especially in the high-incidence settings studied in South and Southeast Asia, and that there is considerable demand for a vaccine against typhoid fever, both among policymakers and community residents in all countries surveyed.

Based on these and other findings, the World Health Organization Scientific Advisory Group of Experts released a draft recommendation for the more widespread use of typhoid vaccines in settings with high typhoid incidence, and both Pakistan and Indonesia will pilot the use of school-based Vi immunization in high-incidence urban settings.

\section{Translational Research on Vaccines after Licensure: Lessons Learned}

Much of the translational research being conducted by the IVI has focused on the third scientific challenge: providing epidemiological, clinical, economic, behavioral and policy evidence for policymakers at both the national and global levels, so that rational decisions can be made about whether to invest in vaccine introduction into public health programs [12]. The IVI has conducted this type of translational research on vaccines against enteric infections, invasive encapsulated bacterial infections, influenza, tuberculosis and flavivirus infections. This research has been done in 22 countries in Asia, Africa and Latin America. In the remainder of the paper, we describe several of the lessons learned from these research studies.

\section{Engaging Policymakers at the Inception of the}

Research

Local policymakers constitute a major audience for the research findings of translational research. Therefore, a major challenge of these programs is to design them in a way that is responsive to the needs of national policymakers in countries for which the findings are intended.

At its inception, the DOMI program conducted a survey of policymakers and opinion leaders in Bangladesh, China, India, Indonesia, Pakistan, Thailand and Vietnam [40]. Overall, approximately 200 face-to-face, semistructured interviews were conducted with personnel in the Ministries of Health, Ministries of Finance and the
National Regulatory Authorities, academic leaders in infectious diseases, and local vaccine producers. Results of these interviews showed that policymakers needed 4 types of information to make decisions about the introduction of new vaccines against cholera, shigellosis and typhoid fever into their public health programs:

(1) acquisition of epidemiological data on the burden and distribution of diseases in their populations;

(2) pilot demonstration projects of licensed vaccines under real-life conditions;

(3) analyses of the economic impact of introducing vaccines into public health programs;

(4) assessments of community and providers' perceptions about the target disease and the demand for a vaccine.

These findings guided the design of the DOMI program and its component translational research studies. The survey also initiated an ongoing dialogue between the DOMI program and policymakers and augmented the impact of the research findings on policy decisions.

\section{Partnerships and Capacity Building}

If translational research is to have an impact on policy, it must be done with broadly inclusive partnerships with all important stakeholders. The DOMI program, for example, successfully created a wide network of partners, including the World Health Organization, Ministries of Health and local investigators in partner countries, international technical experts, and both international and emerging vaccine producers.

An important consideration for accelerated vaccine introduction is conducting the translational research within the existing national research infrastructure of the developing countries for which the research is intended. While reliance on industrialized country researchers and research institutions may be expedient in the short term, research is much more likely to be communicated to national decision makers and to influence policy if it is done 'within the system' and if countries feel ownership of the research. This approach to research has been a cornerstone of the DOMI program.

An essential component of meaningful partnerships with developing country professionals is capacity-building, and the DOMI program made substantial investments into training in the areas of epidemiology, clinical trials, microbiological diagnosis, economic and sociobehavioral research, as well as in vaccine production and regulation. 
Table 3. Annual incidence of Hib meningitis in the 3 IVI field sites, 2000-2002

\begin{tabular}{|c|c|c|c|c|c|c|}
\hline \multirow{3}{*}{$\begin{array}{l}\text { Field } \\
\text { site }\end{array}$} & \multirow{3}{*}{$\begin{array}{l}\text { Population } \\
<5 \text { years old }\end{array}$} & \multicolumn{4}{|c|}{ Hib meningitis patients } & \multirow{3}{*}{$\begin{array}{l}\text { Total Hib } \\
\text { annual } \\
\text { rate, } \times 10^{-5}\end{array}$} \\
\hline & & \multicolumn{2}{|c|}{$\begin{array}{l}\text { CSF culture-confirmed } \\
\text { Hib }\end{array}$} & \multicolumn{2}{|c|}{$\begin{array}{l}\text { CSF PCR or LA }(+) / \\
\text { culture }(-) \mathrm{Hib}^{1}\end{array}$} & \\
\hline & & $\mathrm{n}$ & $\begin{array}{l}\text { annual } \\
\text { rate, } \times 10^{-5}\end{array}$ & $\mathrm{n}$ & $\begin{array}{l}\text { annual } \\
\text { rate, } \times 10^{-5}\end{array}$ & \\
\hline Korea & 116,894 & 8 & 3.4 & 6 & 2.6 & 6.0 \\
\hline Vietnam & 94,529 & 13 & 6.9 & 10 & 5.3 & 12.2 \\
\hline China & 168,060 & 3 & 1.1 & 1 & 0.4 & 1.4 \\
\hline
\end{tabular}

$\mathrm{CSF}=$ Cerebrospinal fluid.

${ }^{1}$ Hib meningitis confirmed by polymerase chain reaction (PCR) or latex agglutination (LA) testing, but negative by culture.

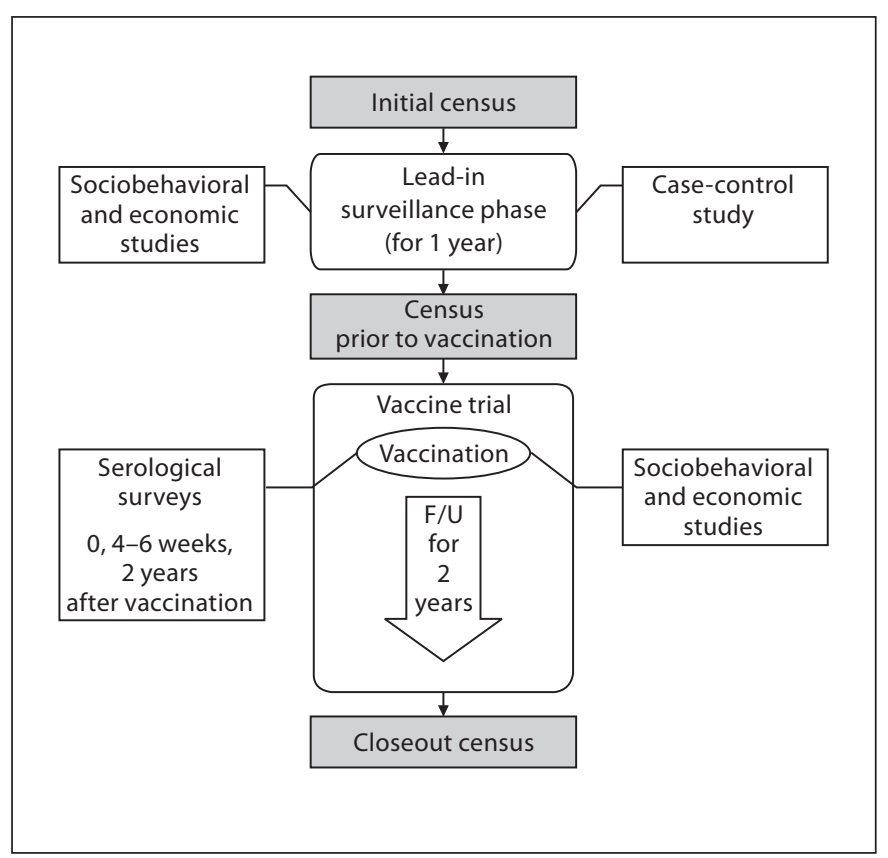

Fig. 2. Structure of the Vi vaccine effectiveness trials of the DOMI program.

\section{Building a Sufficient Evidence Base at the Country Level}

As illustrated by the results of the DOMI policymaker survey, policymakers need diverse, multidisciplinary evidence in their deliberations about introducing new vaccines. This often means that a complete evidence base must be built at the country level. For this reason, the translational research activities of the DOMI program were formulated using a matrix approach. Table 2 shows such a matrix for the translational research of the DOMI program on the $\mathrm{Vi}$ vaccine against typhoid fever.

It was found that an efficient way to produce these data is to nest several types of studies into the same field project. As shown in figure 2, the DOMI program field demonstration projects of the $\mathrm{Vi}$ polysaccharide vaccine against typhoid fever included studies not only of the feasibility, costs, acceptability and protective impact of the $\mathrm{Vi}$ vaccine, but also evaluation of typhoid disease incidence and risk factors, disease costs, as well as population perceptions about typhoid fever and the need for a typhoid vaccine in their programs.

\section{Coordinated Multi-Country Studies}

Isolated studies in a single country can certainly be of value, but coordinated multi-country studies that yield credible comparative data can greatly enhance the value of translational research studies. The DOMI multi-disciplinary typhoid studies shown in figure 2 were done in a standardized, coordinated fashion in field sites in Hechi, China; Jakarta, Indonesia; Hue, Vietnam; Kolkata, India; and Karachi, Pakistan [37, 39]. Overall, these studies enrolled over 200,000 subjects, and comparison of the data from the sites provided several important insights. For example, the studies found a gradient of typhoid incidence rates, with the highest rates in the South Asia sites (Kolkata and Karachi), an intermediate rate in the Southeast Asian site (Jakarta), and the lowest rates in the Northeast Asian sites (Hue and Hechi). In the 3 sites with the 
highest incidence rates (Karachi, Kolkata and Jakarta), typhoid incidence was high not only in school-age children, the age group conventionally thought to be at highest risk of typhoid fever, but also in pre-school-age children, an observation with clear implications for targeting typhoid vaccines [39].

\section{Letting the Chips Fall Where They May}

Translational research studies are typically undertaken by investigators who expect that the study results will motivate vaccine introduction. Nonetheless, it is important to recognize that 'negative' studies, which do not strongly support vaccine introduction, are as important as 'positive' studies. Over the years, the translational research of the IVI has generated both types of data. The first major multi-country research project of the IVI was a study of the burden of Hib meningitis in Asian children. In this prospective study, children $<5$ years old in sites in Korea, China and Vietnam, each with approximately 100,000 children, were followed for 2-year periods to estimate the incidence of Hib meningitis, using common protocols and exhaustive and well-standardized diagnostic methods. This research was undertaken because prospective, population-based studies of Hib meningitis had not been previously done in Asian populations and because of a strong suspicion that Hib meningitis was being underdiagnosed in Asian children, leading to a false impression that this disease was not an important public health problem. To the investigators' surprise, the incidence rates found in the IVI study (table 3 ) were indeed low in each of the 3 sites [41-43].

A contrasting experience was provided by the translational research of the IVI on Japanese encephalitis (JE) in Asian children. Similar to the situation for Hib meningitis, there was a widespread notion that JE was not a significant problem in Asian countries near the Equator, but prospective, population-based studies were lacking. Moreover, there were multiple anecdotal reports of cases of JE by clinicians in Bali, Indonesia. Therefore, IVI investigators, in collaboration with the Ministry of Health of Indonesia, undertook a multi-year, prospective study of JE incidence among all children residing in Bali. Strikingly, the incidence rates found were among the highest reported in Asia, and these data, in conjunction with analyses of potential cost-effectiveness of vaccination against JE in this setting, proved instrumental to a recent decision by the Ministry of Health to introduce routine vaccination against JE for Balinese children [44].

\section{Communication of Results}

Translational research is done to inform policy decisions. Publication of the research in appropriate journals is important. Beyond this, the research must be communicated to policymakers, who may not read these journals, in a timely and efficient fashion. As already noted, engaging policymakers at the outset of the research is helpful in eliciting interest in and continuing dialogue on the research findings as they emerge. Additionally, it is important to communicate the findings in appropriate forums. For example, the DOMI program has held several special symposia and other forums to communicate the findings of the program to policymakers at global and regional levels (WHO and the GAVI Alliance) as well as to national policymakers from the DOMI partner countries. These sessions have been critical to recent policy decisions by Ministries of Health to pilot the introduction of the Vi polysaccharide vaccine against typhoid fever in programs in Indonesia and Pakistan.

\section{Concluding Remarks}

In the initial years since its founding in 1997, the IVI has become a major player in the field of translational research for the introduction of new vaccines into developing countries. While many lessons have already been gleaned from the research of the IVI, and the research itself has had an effect on vaccine policy, improved strategies for designing and conducting programs of translational research are still under development. The recent availability of funding for such programs from the Bill and Melinda Gates Foundation and the GAVI Alliance should increase the effectiveness and efficiency of the research, which is crucial for developing countries whose resources for new vaccines are scarce and for whom correct policy decisions are imperative. 


\section{References}

1 The Jordan report: accelerated development of vaccines 2007. Bethesda, US Department of Health and Human Services, National Institutes of Health, National Institute of Allergy and Infectious Diseases, 2007, p 157.

2 Tacket CO, Kotloff KL, Rennels MB: Initial clinical evaluation of new vaccine candidates: investigators' perspective of phase I and II clinical trials of safety, immunogenicity, and preliminary efficacy; in Levine $\mathrm{M}$, Kaper J, Rappouli R, Liu M, Good M (eds): New Generation Vaccines. New York, Dekker, 2004, pp 19-28.

3 Clemens J, Naficy A, Rao M, Koo H: Longterm evaluation of vaccine protection: methodological issues for Phase III and IV studies; in Levine M, Kaper J, Rappouli R, Liu M, Good M (eds): New Generation Vaccines. New York, Dekker, 2004, pp 29-48.

$\checkmark 4$ Deen J, Clemens J: Issues in the design and implementation of vaccine trials in less developed countries. Nat Rev Drug Discov 2006;5:932-940.

5 Vesikari T, Isolauri E, D’Hondt E, et al: Protection of infants against rotavirus diarrhoea by RIT 4237 attenuated bovine rotavirus strain vaccine. Lancet 1984;i:977-981.

-6 Hanlon P, Hanlon L, Marsh V, et al: Trial of an attenuated bovine rotavirus vaccine (RIT 4237) in Gambian infants. Lancet 1987;i: 1342-1345.

7 De Mol P, Zissis G, Butzler JP, et al: Failure of live, attenuated oral rotavirus vaccine. Lancet 1986;ii:108.

-8 Eskola J, Käyhty H, Takala AK, et al: A randomized, prospective field trial of a conjugate vaccine in the protection of infants and young children against invasive Haemophilus influenzae type b disease. N Engl J Med 1990;323:1381-1387.

-9 Ward J, Brenneman G, Letson GW, et al: Limited efficacy of Haemophilus influenzae type b conjugate vaccine in Alaska native infants. N Engl J Med 1990;323:1393-1401.

10 Mulholland K, Hilton S, Adegbola R, et al: Randomised trial of Haemophilus influenzae type-b tetanus protein conjugate for prevention of pneumonia and meningitis in Gambian infants. Lancet 1997;349:11911197.

11 Clemens J, Brenner R, Rao M, et al: Evaluating new vaccines for developing countries: efficacy or effectiveness? JAMA 1996;275: 390-397.

12 Clemens J, Jodar L: Introducing new vaccines into developing countries: obstacles, opportunities, and complexities. Nat Med 2005;11(suppl 4):S12-S15.

13 Mahoney RT, Maynard JE: The introduction of new vaccines into developing countries. Vaccine 1999;17:646-652.
14 Vesikari T, Matson DO, Dennehy P, et al: Rotavirus Efficacy and Safety Trial (REST) Study Team. Safety and efficacy of pentavalent human-bovine (WC3) reassortant rotavirus vaccine. N Engl J Med 2006;354:2333.

15 Qadri F, Chowdhury MI, Faruque S, et al: Randomized controlled study of the safety and immunogenicity of Peru-15, a live attenuated oral vaccine candidate for Vibrio cholerae $\mathrm{O} 1$ in adult volunteers in Bangladesh. J Infect Dis 2005;192:573-579.

16 Qadri F, Chowdhury M, Faruque S, et al: Peru-15, a live attenuated oral cholera vaccine is safe and immunogenic in Bangladeshi toddlers and infants. Vaccine 2007;25:231238.

17 Hindle Z, Chatfield SN, Phillimore J, et al: Characterization of Salmonella enteritica derivatives harboring defined aroC and Salmonella pathogenicity island 2 type III secretion system (ssaV) mutations by immunization of healthy volunteers. Infect Immun 2002;70:3457-3467.

18 Trach DD, Cam PD, Ke NT, et al: Investigations of the safety and immunogenicity of a new, killed oral cholera vaccine developed in Vietnam. Bull World Health Organ 2002;80: $2-8$.

19 Lin FY, Ho VA, Khiem HB, et al: The efficacy of a Salmonella typhi Vi conjugate vaccine in two-to-five-year-old children. N Engl J Med 2001;344:1263-1269.

20 Crump JA, Luby SP, Minta ED: The global burden of typhoid fever. Bull World Health Organ 2004;82:346-353.

21 Sur D, von Seidlein L, Manna B, et al: The malaria and typhoid fever burden in the slums of Kolkata, India: data from a prospective community-based study. Trans Royal Soc Trop Med Hyg 2006;100:725733.

22 Bahl R, Sinha A, Poulos C, et al: Costs of illness due to typhoid fever in an Indian urban slum community: implications for vaccination policy. J Health Popul Nutr 2004;22: 304-310.

-23 Zhou WZ, Koo HW, Wang XY, et al: Revaccination with locally-produced Vi typhoid polysaccharide vaccine among Chinese school-aged children: safety and immunogenicity findings. Pediatr Infect Dis J 2007;26: 1001-1005.

-24 Acosta CJ, Hong-Hui Y, Ning W, et al: Efficacy of a locally produced, Chinese Vi polysaccharide typhoid fever vaccine during six years of follow-up. Vaccine 2005;23:56185623.

25 Chen X, Stanton B, Pach A, et al: Adult perceived prevalence of enteric fever predicts laboratory-validated incidence of typhoid fever in children. J Health Popul Nutr, in press.
26 Kaljee LM, Pham V, Son ND, et al: Trial participation and vaccine desirability for $\mathrm{Vi}$ polysaccharide typhoid fever vaccine in Hue City, Viet Nam. Trop Med Int Health 2007; 12:25-36.

27 Yang HH, Kilgore PE, Yang LH, et al: An outbreak of typhoid fever, Xing-An county, People's Republic of China, 1999: estimation of the field effectiveness of Vi polysaccharide typhoid vaccine. J Infect Dis 2001;183:17751780.

28 Acosta C, Galindo C, Ochiai RL, et al: The role of epidemiology in the introduction of Vi polysaccharide vaccines in Asia. J Health Popul Nutr 2004;22:240-245.

29 Poulos C, Bahl R, Whittington D, et al: A benefit-cost analysis of typhoid fever immunization programs in an Indian urban slum community: incorporating private market provision of Vi vaccines. J Health Popul Nutr 2004;22:311-321.

30 Yang J, Acosta C, Si G, et al: A mass vaccination campaign targeting adults and children to prevent typhoid fever in Hechi; expanding the use of $\mathrm{Vi}$ polysaccharide in Southeast China: a cluster randomized trial. BMC Public Health 2005; 18:49-55.

- 31 Ochiai RL, Wang XY, Yang J, et al: A relative increase in Salmonella paratyphi A rates in Asia: results from surveillance studies in 5 countries. Emerg Infect Dis 2005;11:17641766.

32 Acosta C, Galndo C, Ali M, et al: A multicountry cluster randomized controlled effectiveness evaluation to accelerate the introduction of $\mathrm{Vi}$ polysaccharide typhoid vaccine in developing countries in Asia: rationale and design. Trop Med Int Health 2005; 10:1219-1228.

33 Khan MI, Sahito SM, Khan MJ, et al: Enhanced disease surveillance through private health care sector cooperation in Karachi, Pakistan: experience from a vaccine trial.

Bull World Health Organ 2006;84:72-77.
Khan MI, Ochiai L, Hanza H, et al: Lessons and implications from a mass immunization campaign in squatter settlements of Karachi, Pakistan: an experience from a cluster-randomized, double-blind controlled clinical trial. Trials 2006;7:17.

-35 Thiem V, Donovaro-Holliday C, Canh do G, et al: The feasibility of a school-based Vi polysaccharide vaccine mass immunization campaign in Hue City, central Vietnam: streamlining a typhoid fever preventive strategy. Southeast Asian J Trop Med Public Health 2006;37:515-522. 
>36 Agtini M, Ochiai RL, Soeharno R, et al: Introducing $\mathrm{Vi}$ polysaccharide typhoid vaccine to primary school children in North Jakarta, Indonesia, via an existent school-based vaccination platform. Public Health 2006; 120:1081-1087.

-37 Ochiai RL, Acosta C, Agtini M, et al: The use of typhoid vaccines in Asia: the DOMI experience. Clin Infect Dis 2007;45(suppl 1):S34S38.

-38 Sur D, Ali M, von Seidlein L, et al: Comparisons of predictors for typhoid and paratyphoid fever in Kolkata, India. BMC Public Health 2007;7:289.
39 Ochiai RL, Acosta C, Danovaro-Holliday MC, et al: A multicenter, population-based, prospective surveillance study of typhoid fever in 5 Asian countries: disease burden and implications for control. Bull World Health Organ, in press.

40 DeRoeck D, Nyamete A, Mahoney R, Clemens J: Policymakers' views regarding the introduction of new generation vaccines against cholera, typhoid fever and shigellosis in Asia. Vaccine 2005;23:2762-2774.

41 Anh DD, Kilgore P, Kennedy W, et al: Haemophilus influenzae type $\mathrm{b}$ meningitis among children in Hanoi, Vietnam: epidemiologic patterns and estimates of disease burden. Am J Trop Med Hyg 2006;74:509515.
42 Kennedy W, Chang S, Purdy K, et al: Incidence of bacterial meningitis in Asia using enhanced CSF testing: polymerase chain reaction, latex agglutination, and culture. Epidemiol Infect 2007;5:1-10.

43 Kim JS, Jang YT, Kim JD, et al: Incidence of Haemophilus influenzae type b and other invasive diseases in South Korean children. Vaccine 2004;28:3952-3962.

44 Kari K, Liu W, Gautama K, et al: A hospitalbased surveillance for Japanese encephalitis in Bali, Indonesia. BMC Med 2006;4:8. 